\title{
Application of Big Data in Strengthening Livestock Service Delivery System
}

\author{
Shreyansh Hosure* and K. Anjali \\ Department of Veterinary and Animal Husbandry Extension, CoVAS, Mannuthy, India \\ *Corresponding author
}

\section{A B S T R A C T}

\section{Keywords}

Big Data, livestock, Service delivery

Article Info

Accepted:

18 May 2020

Available Online:

10 June 2020
Technology enhancement has made the evolution of Big Data. Big Data refers to large sets of complex data, both in structured and unstructured form which traditional processing techniques and algorithms are unable to operate on it. The characteristics of big data has five 'Vs' viz., volume, velocity, veracity, value and variety. Big Data has a long way to go in the veterinary sector, we can already see some impacts it has made for animals across the globe. The innovation and spread of big databases is slowly picking up with creations like VetCloud, PigWise, VETport, Life Learn and many others contributing to this innovation. National Dairy Development Board (NDDB) is harnessing big data for assessing the nutrition requirement for milch animals. RFID delivers big data on cows and milk which helped in increasing the milk yield up to 5 liters. The big data revolution is already having an influence on dairy industry, as it has in other sectors. The timing is currently ideal for the veterinary profession to ensure that they are at the forefront of these developments, taking the opportunities created by big data and helping farmers maximize value from investment in technology.

\section{Introduction}

Nowadays, companies and industries are starting to realize the importance of data availability in large amounts in order to make the right decisions and support their strategies. With the development of new technologies, the internet and social networks, the production of digital data is constantly growing. The digital data produced is partly the result of the use of devices connected to the internet called IoT (Internet of Things). Thus smart phones, tablets and computers transmit data about their users. Connected smart objects convey information about consumer's use of everyday objects (Youssra and Sara, 2018). Apart from the connected devices, data come from a wide range of sources demographic data, climate data, scientific and medical data and energy consumption data.

All these data provide information about the location of users of the devices, their travel, their interests, their consumption habits, their leisure activities and their projects and so on. But also information on how the infrastructure, machinery and apparatus are 
used. With the ever-increasing number of internet and mobile phone users, the volume of digital data is growing rapidly (Batistic and Laken, 2019).

\section{Big data}

The term first used in 1990. Includes huge amount of data sets and it is not in organised form. We need to handle it carefully and uncover the hidden patterns of it (Steven Sonka, 2016).

The term Big Data refers to the evolution and use of technologies that provide the right user at the right time with the right information from a mass of data that has been growing exponentially for a long time in our society.

The challenge is not only to deal with rapidly increasing volumes of data but also the difficulty of managing increasingly heterogeneous formats as well as increasingly complex and interconnected data (Youssra and Sara, 2018).

Big Data refers to large sets of complex data, both structured and unstructured form which traditional processing techniques and algorithms are unable to operate on it. It aims to reveal hidden patterns and has led to an evolution from a model-driven science paradigm into a data-driven science paradigm (Ajit Kumar, 2016).

Big data often means dirty data as fraction of data inaccuracy is increases with data volume growth. Human inspection at big data scale is impossible and there is desperate need for intelligent tools of accuracy and believability control.

One-third of the globally stored information is in alphanumeric, still images, video and audio content to analyse this huge data we require Big Data tools (Mirkes et al., 2016).

\section{Characteristics of big data}

These are the 5Vs of Big Data.

\section{Volume}

It represents the amount of data generated, stored and operated within the system. The increase in volume is explained by the increase in the amount of data generated and stored, but also by the need to exploit it.

\section{Variety}

It represents the multiplication of the types of data managed by an information system. This multiplication leads to a complexity of links and link types between these data. The variety also relates to the possible uses associated with a raw data.

\section{Velocity}

It represents the frequency at which data is generated, captured and shared. The data arrive by stream and must be analysed in real time.

\section{Veracity}

It represents the level of quality, accuracy and uncertainty of data and data sources.

\section{Value}

It represents the value and potential derived from data (Taylor-Sakyi, 2016).

\section{Big data in strengthening livestock service delivery system}

\section{National dairy development board (NDDB) harnessing big data}

Under Ration Balancing Programme, local resource person formulates a least cost 
balanced ration for milch animals from locally available feed resources using the software "Information Network for Animal Productivity and Health (INAPH)". Balanced ration to milch animals helps in ensuring that the milch animals produce milk commensurate with their genetic potential. Feeding the balanced ration to milch animals not only reduces the cost of feeding per $\mathrm{Kg}$ of milk but also significantly reduces methane emissions (Annual Report, 2017).

\section{Pig wise - web based platform}

PigWise as an example, hog farms have used this web based platform to virtually share herd health and disease data with veterinarians. Sharing this has already helped maximize the reproductive performance of the pigs, prevent extremely contagious respiratory diseases on their farms and understands the transmission path of new diseases being spread.

User-friendly is the name of the game with PigWise. After an account is created through the local Zoetis representative free of charge, managers or owners can assign a name to each farm, authorize users, create batches for groups of hogs and set thresholds for tracking important health metrics.

From there, the pig caregivers can use the PigWise app on a smartphone to enter daily information for a group of hogs starting from the time the pigs enter the facility. On a basic level, each farm can create customized fields to track daily records like hog inventory, receiving feed, barn temperatures, water intakes, mortalities and medical treatments. Employees can identify sick pigs and use a dropdown menu to classify symptoms, log treatment and document other essential information. PigWise also can work in conjunction with individual pig care training from Zoetis, so caregivers can enter information about sick pigs based on the $\mathrm{A}$, $\mathrm{B}, \mathrm{C}$ or E classification system.

As soon as the information is entered into the fields on the mobile app and synchronized with the dashboard, that data is readily available for review. Production managers and veterinarians can get access anytime to all information entered by caregivers by logging into the app on a Smartphone or Web-based dashboard. Upon login, managers and veterinarians will see two options: batches for review and items that need attention. The application records daily metrics on temperature, water consumption and mortalities. Once the metrics fall into a red zone based on established thresholds, it will alert managers and veterinarians. Those alerts allow for quicker interventions to improve pig health and performance (Bargmann, et al., 2016).

\section{RFID delivers big data on cows and milk}

The Indian company, Chitale Dairy, has gone a similar route by increasing the overall quality of milk production from their cows by having Radio Frequency Identification Tags (RFID) placed on each cow to transmit information to the cloud and mobile devices. In order for farmers to do everything that is instructed correctly, "the farm then sends a todo list to farmers in their local language each morning on what each cow needs based on the data collected from the RFID signals". The execution of this allows farmers to do everything needed which will also inform them of any warning signs a cow may give off indicating illness.

The spread of Big Data has also surprisingly helped increase the milk yield from cows in India. Each cow on this farm has a tag that will send their health information to their owner such as their blood profile, whether or not they are in heat, need to be vaccinated, 
have proper nutrition and so much more. Having all of these features in their system has helped increase the lifespan and milk production of their cows (Wankhede and Pednekar, 2016).

\section{Big data helps in protecting of endangered species}

The Vertica Platform, designed by HP to provide early warning signs amongst animal numbers who are at risk of extinction. The system collects data from thousands of cameras and climate sensors and uses the data to collate information on population numbers. This information is then fed into the big data platform, which allows for fast and accurate readings that help target specific species or areas. Due to the large quantity of images, it was almost impossible to manage.

Researchers would spend weeks, sometimes even months analyzing the data, making collations and then drawing conclusions. Due to the time sensitive nature of the work, if the endangered population is found too late, it could mean the end for this species.

By the big data platform provided by HP, processing speed has almost doubled, thus providing an effective early warning system and by doing so, freed up a lot of time for the personnel to actual make a difference and act on the data received (Lewis, et al., 2018).

\section{Vet compass australia program}

The Vet Compass Australia program collects real-time clinical records from veterinary practices and aggregates them for researchers to interrogate. It delivers Australian researchers sustainable and cost-effective access to authoritative data from hundreds of veterinary practitioners. Effective data linkage of clinical records for the development of spatial epidemiological tools is needed to quantify the geographical limits of infectious disease occurrence, the populations at risk and to identify major risk factors for zoonotic infections of significance to public health. Vet Compass Australia will provide the evidence to allow refinement of existing clinical protocols with the aim of implementing targeted control and mitigation strategies to directly decrease animal disease burden.

Companion-animal research is under-funded in Australia, with the chief sources of funding being confined to small non-profit organizations, pet-food manufacturers and pharmaceutical manufacturers who, quite understandably, have partisan research needs.

This can impede long-term, large-scale studies into the burden of disease, risk factors and cost-effectiveness of treatments for companion animals. The merit of individual therapeutic agents may differ with chronicity and even breed, so it seems clearly beneficial to collect Big Data that may allow clinical guidelines for any disorders of companion animals to be fine-tuned as more evidence becomes available (McGreevy, et al., 2017).

\section{National surveillance systems - Newzealand}

It provides an example of a prototype to capture data from electronic medical records for use in national surveillance systems and clinical research in New Zealand. Clinical data and diagnoses from a subset of 344 patient consults were extracted from two veterinary clinics and incorporated into a dedicated database to be analyzed at the population level.

Companion animal and equine veterinary practitioners were asked to test the feasibility of this national practice-based health information and data system and to identify strategies that would increase practitioners engagement. The geospatial integration of 
data, for example, would help to establish the baseline incidence of companion animal and equine disease in New Zealand, detect anomalies that may suggest an emerging disease threat or welfare issue, improve disease management, support research activities and inform policy (Muellner, et al., 2016).

\section{Disease watch dog surveillance website- Austaralia}

This study demonstrates that internet search metrics can be used to monitor the occurrence of tick paralysis in companion animals, which would facilitate early detection of high-risk periods for tick paralysis cases. It constitutes the first application of the rapidly emerging field of Internet-based surveillance to veterinary science.

Monitoring and early detection of high risk period for tick paralysis cases in companion animals. Metrics data were available for 24 relevant search terms at national level, 16 for QLD and 18 for NSW and they were all significantly correlated with tick paralysis notifications.

Monthly update will be given for registered users. Monthly reports of tick paralysis cases were obtained for a three year period form Queensland (QLD) and New South Wales (NSW) - reports $98 \%$ of cases. Resulting 8,414 tick paralysis cases were registered (Guernier, et al., 2016).

Technology enhancement has made the evolution of Big Data. Even though it is dirty data it can be used for many purposes. The availability of Big Data, low-cost commodity hardware and new information management and analytic software has produced a unique moment in the history of data analysis. The convergence of these trends means that we have the capabilities required to analyse astonishing data sets quickly and costeffectively for the first time in history. The big data revolution is already having an influence on the veterinary field, as it has in other sectors. The timing is currently ideal for the veterinary profession to ensure that they are at the forefront of these developments, taking the opportunities created by big data and helping farmers maximize value from investment in technology.

\section{References}

Ajit Kumar, R. 2016. Impact of Big Data Analytics on Healthcare and Society. Journal of Biometrics and Biostatistics, 7: 2.

Annual Report, 2017. [book on-line]. National Dairy Development Board. Available:

http://dahd.nic.in/sites/default/filess/ND DB_AR_2017_Eng.pdf [28 April. 2020].

Bargmann, S., Long, M., Kerner, J., Fagan, J.M. 2016. Big Data can be expected to Advance Veterinary Medicine. Journal of Veterinary Medicine, 15: 225-240.

Batistic, S. and Laken, V. 2019. History, Evolution and Future of Big Data and Analytics: A Bibliometric Analysis of Its Relationship to Performance in Organizations. British Journal of Management, 30: 229-251.

Guernier, V., Milinovich, G.J., Santos, M.A.B., Haworth, M., Coleman, G. and Magalhaes, R.J.S. 2016. Use of big data in the surveillance of veterinary diseases: early detection of tick paralysis in companion animals. Parasite. Vector, 9: 303.

Lewis, K.P., Vander Wal, E. and Fifield, D.A. 2018. Wildlife biology, big data, and reproducible research. Wildlife Society Bulletin, 42: 172-179.

McGreevy, P., Thomson, P., Dhand, N., Raubenheimer, D., Masters, S., 
Mansfield, C., Baldwin, T., Soares Magalhaes, R., Rand, J., Hill, P. and Peaston, A. 2017. Vet Compass Australia: a national big data collection system for veterinary science. Animals, 7: 74

Mirkes, E.M., Coats, T.J., Levesley, J. and Gorban, A.N. 2016. Handling missing data in large healthcare dataset: A case study of unknown trauma outcomes. Computers in Biology and Medicine, 75: 203-216.

Muellner, P., Muellner, U., Gates, M.C., Pearce, T., Ahlstrom, C., O’Neill, D., Brodbelt, D. and Cave, N.J. 2016. Evidence in practice-a pilot study leveraging companion animal and equine health data from primary care veterinary clinics in New Zealand Frontiers in Veterinary Science, 3: 116. Steven Sonka. 2016. Big Data Characteristics. International Food and Agribusiness Management, 19: 7-12.

Taylor-Sakyi, K. 2016. Big data: Understanding big data. arXiv preprint arXiv:1601.04602.

Wankhede, K. and Pednekar, S. 2016. Animal Tracking and Caring using RFID and IOT. Journal of Computational Design and Engineering, 56: 24-27.

Youssra, R. and Sara, R. 2018. Big data and big data analytics: concepts, types and technologies. International Journal of Research and Engineering, 5: 524-528.

\section{How to cite this article:}

Shreyansh Hosure and K. Anjali. 2020. Application of Big Data in Strengthening Livestock Service Delivery System. Int.J.Curr.Microbiol.App.Sci. 9(06): 1383-1388.

doi: https://doi.org/10.20546/ijcmas.2020.906.172 